\title{
reviscafuences
}

ISSN: 1575.7072 | 8.15SN: 2172.7775

Páginas: 150-162

Recibido: 2020-09-28

Revisado: 2020-11-13

Aceptado: 2021-02-12

Preprint: 2021-05-10

Publicación Final: 2021-05-15

\section{Cuestionario sobre conocimientos del profesorado universitario de matemáticas para la integración tecnológica}

\section{Questionnaire on knowledge of university mathematics teachers for technological integration}

\author{
(iD) Frank Alain Castro Sierra \\ Universidad de las Ciencias Informáticas (Cuba) \\ (iD) Elba Gutierrez-Santiuste \\ Universidad de Granada (España)
}

\begin{abstract}
En esta investigación se desarrolla un cuestionario de autoevaluación para medir el conocimiento del profesorado universitario de Matemática sobre la integración tecnológica en las aulas. El modelo Technological Pedagogical Content and Knowledge (TPACK) es reconocido por la comunidad científica para describir la comprensión de los docentes sobre la compleja interacción entre contenido, pedagogía y tecnología. Se adapta el cuestionario de Schmidt et al. (2009) al ámbito de las Matemáticas. El cuestionario está compuesto por 28 ítems y se evalúan mediante una escala Likert de cuatro niveles (1: nunca; 4: siempre). La muestra fue de 183 docentes universitarios de Matemáticas. El análisis de modelos de ecuaciones estructurales se utilizó para examinar los factores del modelo TPACK. Los resultados confirman el modelo de siete factores e indican que el TPACK percibido por el profesorado se correlaciona de manera positiva con todos los demás factores. Finalmente, se concluye que el instrumento es válido y fiable.

\section{Abstract}

The aim of this research is to develop a self-evaluation questionnaire to measure the knowledge of university mathematics teachers about technological integration in the classroom. The TPACK model is recognized by the scientific community to describe teachers' understanding of the complex interaction between content, pedagogy and technology. The Schmidt et al. (2009) questionnaire is adapted to the field of Mathematics. The questionnaire is made up of 28 items and is assessed using a Likert scale of four levels (1: never; 4: always). The sample was 183 university teachers of Mathematics. The analysis of structural equation models was used to examine the factors of the TPACK model. The results confirm the seven factors model and indicate that the TPACK perceived by teachers correlates positively with all other factors. Finally, it is concluded that the instrument is valid and reliable.
\end{abstract}

\section{Palabras clave}

Educación superior, Matemáticas, Cuestionario, Tecnología de la información, Profesor, Tecnología educativa, Competencias del docente, Conocimiento.

\section{Keywords}

Higher education, Mathematics, Questionnaires, Information technology, Teachers, Educational technology, Teacher qualifications, Knowledge.

\section{Introducción}

Con el vertiginoso desarrollo de la tecnología en los últimos años se ha reconocido que el profesorado debe estar preparado para integrar adecuadamente las Tecnologías de la Información y la Comunicación (TIC) 
en su práctica educativa. Aplicar la tecnología para maximizar la efectividad en la enseñanza, requiere del profesorado suficiente conocimiento pedagógico, del contenido de la materia y tecnológico. En concordancia, numerosos estudios se han orientado en el modelo Technological Pedagogical Content and Knowledge (TPACK) para ayudar al profesorado a lograr mejores resultados de enseñanza y aprendizaje (Baser et al., 2016; Chua et al., 2017; Tseng, 2018; Zhou y Jin, 2017).

A medida que los académicos han propuesto una nueva visión sobre el conocimiento docente requerido para la enseñanza en el siglo XXI, van surgiendo muchas preguntas (Niess, 2011). Estas cuestiones se focalizan en: a) cómo y cuándo incorporar las tecnologías para la enseñanza y el aprendizaje de diversas disciplinas y b) qué experiencias y preparación son esenciales para desarrollar el conocimiento pedagógico y guiar el aprendizaje en las diversas áreas de estudio con tecnologías digitales. Las respuestas pueden ser observadas en las instituciones educativas donde el docente debe tener las competencias necesarias para integrar las TIC en la Educación. Evidencias empíricas han mostrado que el profesorado universitario generalmente no tiene la preparación adecuada para incorporar las TIC en las aulas (Agyei y Voogt, 2015; Finger et al., 2015; Jang y Tsai, 2013) y, por consiguiente, varias instituciones han realizado acciones encaminadas a desarrollar los conocimientos y habilidades del profesorado para integrar las TIC.

En relación a la problemática expuesta, el modelo TPACK ha sido construido basado en las aportaciones de Shulman (1986, 1987), el cual subrayaba la importancia del conocimiento pedagógico y del contenido que debía poseer un docente para impartir clases. Este autor define el conocimiento pedagógico del contenido (PCK) como la mezcla de contenido de la materia a impartir y la pedagogía para el entendimiento de cómo los temas o problemas se organizan, representan y adaptan a los diversos intereses del alumnado. Igualmente, el modelo TPACK se fundamenta en el estudio de Mishra y Koehler (2006) con el objetivo de describir la comprensión por parte de los docentes sobre la compleja interacción entre contenido, pedagogía y tecnología. La base del modelo TPACK está formada por tres componentes principales de conocimientos según Koehler et al. (2014):

- Conocimiento del contenido (CK) se refiere al conocimiento que debe tener el profesorado sobre la materia que debe impartir.

- Conocimiento pedagógico (PK) considera al conocimiento que posee el profesorado sobre prácticas de enseñanza, estrategias y métodos para promover el aprendizaje del alumnado.

- Conocimiento de la tecnología (TK) apunta al conocimiento del profesorado sobre las tecnologías tradicionales y nuevas que pueden integrarse en la docencia y en el aprendizaje.

La interacción de los componentes principales del modelo origina otros cuatro componentes:

- Conocimiento tecnológico del contenido (TCK) asocia al conocimiento sobre cómo puede ser representado el conocimiento de la materia con las tecnologías. Este conocimiento incluye la capacidad de utilizar la tecnología disponible para contenidos de la materia.

- Conocimiento pedagógico del contenido (PCK) es la noción de Shulman (1986) de "una comprensión de cómo los temas o problemas particulares se organizan, representan y adaptan a los diversos intereses y habilidades de los alumnos, y se presentan para la instrucción.” (p. 8)

- Conocimiento tecnológico pedagógico (TPK) corresponde al conocimiento sobre cómo usar las tecnologías en las prácticas de enseñanza, estrategias y métodos para promover el aprendizaje del alumnado. Este conocimiento incluye la capacidad de comprender que la tecnología puede cambiar la enseñanza.

- El conocimiento tecnológico del contenido pedagógico (TPACK) vincula "al conocimiento sobre las complejas relaciones entre tecnología, pedagogía y contenido que permiten a los docentes desarrollar estrategias de enseñanza apropiadas y específicas al contexto." (Koehler et al., 2014, p. 102)

La noción de TPACK ha ganado reconocimiento y aceptación por parte de los investigadores educativos en los últimos años. Algunas publicaciones realizadas sobre este modelo, en el período de 2002-2016, han sido analizadas en Chai et al. (2013), Voogt et al. (2013), Wu (2013) y Willermark (2018), desde los siguientes indicadores: (a) fuente de publicación, (b) enfoques de investigación, (c) áreas de conocimientos y (d) grupos de muestra.

Las investigaciones de Chai et al. (2013) y Willermark (2018) coinciden en que más del 55\% de las contribuciones sobre TPACK están publicadas en revistas de Tecnología Educativa. Hasta el año 2011 han sido realizadas en su mayoría con enfoques de investigación cualitativos o cuantitativos, no comportándose así con enfoques mixtos (Chai et al., 2013; Wu, 2013). Desde el año 2011 y hasta el 2016 los enfoques cuantitativos y mixtos tienen más del $87 \%$ de publicaciones, mientras el cualitativo tiene un $12 \%$ (Willermark, 2018). Se han dirigido hasta el año 2016 las contribuciones científicas en la temática a diferentes disciplinas 
(ej. Ciencias, Matemáticas, Ciencias Sociales, Geografía, etc.), disminuyendo en la disciplina Matemática el número de investigaciones (Chai et al., 2013; Willermark, 2018; Wu, 2013). Los grupos a quienes se han dirigido las investigaciones sobre TPACK en la actualidad son generalmente al profesorado en formación y en servicio.

Varios han sido los enfoques asumidos por los investigadores para abordar la incorporación de las TIC por el profesorado, haciendo uso del modelo TPACK. La revisión realizada por Voogt et al. (2013) sobre este modelo, identificó que las investigaciones se dirigen a: entendimiento y/o avance del modelo, la medición del TPACK, y/o estrategias de desarrollo TPACK del profesorado. La medición y desarrollo de TPACK en el profesorado requiere de disímiles instrumentos para triangular información. Actualmente el enfoque más común para identificar el TPACK según Willermark (2018) son los cuestionarios de autoevaluación.

Debe señalarse que se han realizado instrumentos (Chai et al., 2010; Cubeles y Riu, 2018; Kaya y Dağ, 2013; Koehler y Mishra, 2005; Koh et al., 2010; Sahin, 2011; Schmidt et al., 2009; Valtonen et al., 2017) encargados de la autoevaluación de los conocimientos del profesorado. Por ejemplo, Schmidt et al. (2009) desarrollaron un instrumento para medir las percepciones de autoeficacia del profesorado en formación. De ahí que el instrumento se diseñara para los siete componentes del modelo, con 75 ítems inicialmente y una escala Likert de cinco niveles (1: muy en desacuerdo; 5: totalmente de acuerdo). La validez del contenido fue revisada por 3 expertos en TPACK. El Análisis Factorial Exploratorio (AFE) condujo a los siete factores $(n=124)$ del modelo con un total de 47 ítems. El instrumento tiene como limitación que los constructos PCK y TCK incluyeron cuatro ítems cada uno, y cada ítem se conectaba con un área de contenido (matemáticas, literatura, ciencias y estudios sociales).

Si bien es cierto que investigaciones como la de Schmidt et al. (2009) se han desarrollado para esclarecer la teoría del modelo TPACK, también es válido apuntar que este instrumento no permite a los investigadores extraer implicaciones más específicas para el desarrollo profesional docente. De ahí que los investigadores Chai, Koh y Tsai (2016) identificaran la necesidad de desarrollar cuestionarios para tecnologías, pedagogías y contenidos específicos.

Cabe considerar, en el caso de los cuestionarios para medir TPACK sobre contenidos específicos (Bilici et al., 2013; Chai et al., 2013; Graham et al., 2009; Jang y Tsai, 2013; Lin et al., 2013), las recomendaciones que realizan Chai et al. (2016) sobre una forma de diseñarlos a partir de cuestionarios generales validados. De ahí que los autores identifiquen dentro de las futuras direcciones para investigar: a) la creación de cuestionarios para contextos específicos tecnológicos, pedagógicos y de contenido, y b) la evaluación del TPACK del profesorado en entornos de enseñanza y aprendizaje de la Educación Superior.

La presente investigación tiene por objetivo desarrollar un instrumento de autoevaluación para medir el conocimiento técnico, pedagógico y tecnológico del profesorado universitario de Matemática. El cuestionario que se proporciona, se distingue de otros en las siguientes características:

1. Es una adaptación del trabajo realizado por Schmidt et al. (2009) dirigido a desarrollar un cuestionario extensible a diferentes contextos, en el área específica de las Matemáticas.

2. Este estudio se basa en las contribuciones del profesorado universitario en servicio intentando aportar un cuestionario de calidad para evaluar el TPACK.

\section{Metodología}

Se utilizó un muestreo por criterio para seleccionar participantes en base a características predeterminadas, específicamente, profesorado que actualmente imparte clases de Matemáticas en la universidad. El cuestionario se publicó de forma online en febrero de 2018, a 1017 docentes universitarios de Matemáticas. Se recopilaron un total de 183 respuestas de seis países diferentes, lo que representa una tasa de respuesta general del $17.9 \%$. Los descriptivos de los participantes se muestran en la Tabla 1.

En la revisión sobre las medidas cuantitivas del TPACK realizada por Chai et al. (2016), los cuestionarios han sido la medida más utilizada, fueron clasificados por los autores en cuatro grupos para una mejor comprensión: a) generales, b) especializados para tecnologías, c) especializados para pedagogías y d) especializados para áreas de contenidos específicos. En la Tabla 2 se expone información sobre algunos cuestionarios de medición del TPACK, los cuales se clasifican según los criterios de Chai et al. (2016). El 46.15\% de los cuestionarios revisados se clasifican como generales, entre ellos se constata como característica que la mayoría son adaptaciones de Schmidt et al. (2009).

Schmidt et al. (2009) realizan un análisis factorial exploratorio (AFE) donde identifican los 7 factores del modelo TPACK en un cuestionario diseñado específicamente para profesores en formación de educación primaria o de educación infantil. El dominio del conocimiento del contenido incluye ítems específicos para las áreas de matemáticas, ciencias, estudios sociales y literatura. Se adaptará este cuestionario general, que demostró 
Tabla 1

Descriptivos de los participantes

\begin{tabular}{|c|c|c|c|}
\hline $\begin{array}{l}\text { Variable } \\
\text { Género }\end{array}$ & Número (\%) & & M (SD) \\
\hline Femenino & 76 & $(41.5)$ & 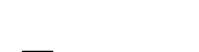 \\
\hline $\begin{array}{l}\text { Masculino } \\
\text { Grado académico } \\
\text { o científico }\end{array}$ & 107 & $(58.5)$ & \\
\hline Doctor & 118 & (64.5) & \\
\hline Máster & 43 & (23.5) & \\
\hline Años Experiencia docente & & & $22.86(13.42)$ \\
\hline 1 a 5 años & 21 & (11.5) & \\
\hline 6 a 10 años & 27 & (14.8) & \\
\hline 11 a 15 años & 20 & (10.9) & \\
\hline 16 o más años & 115 & (62.8) & \\
\hline Años Experiencia con TIC & & & $12.69(8.46)$ \\
\hline 1 a 5 años & 34 & (18.6) & \\
\hline 6 a 10 años & 36 & (19.7) & \\
\hline 11 a 15 años & 40 & (21.9) & \\
\hline 16 o más años & 73 & (39.9) & \\
\hline
\end{tabular}

Nota. M: valor medio; SD: desviación estándar

tener confiabilidad y validez, teniendo en cuenta las consideraciones dadas por Chai et al. (2016) sobre la forma de desarrollar cuestionarios TPACK de contenidos específicos, a partir de cuestionarios TPACK generales validados.

\section{Tabla 2}

Cuestionarios de medición del TPACK

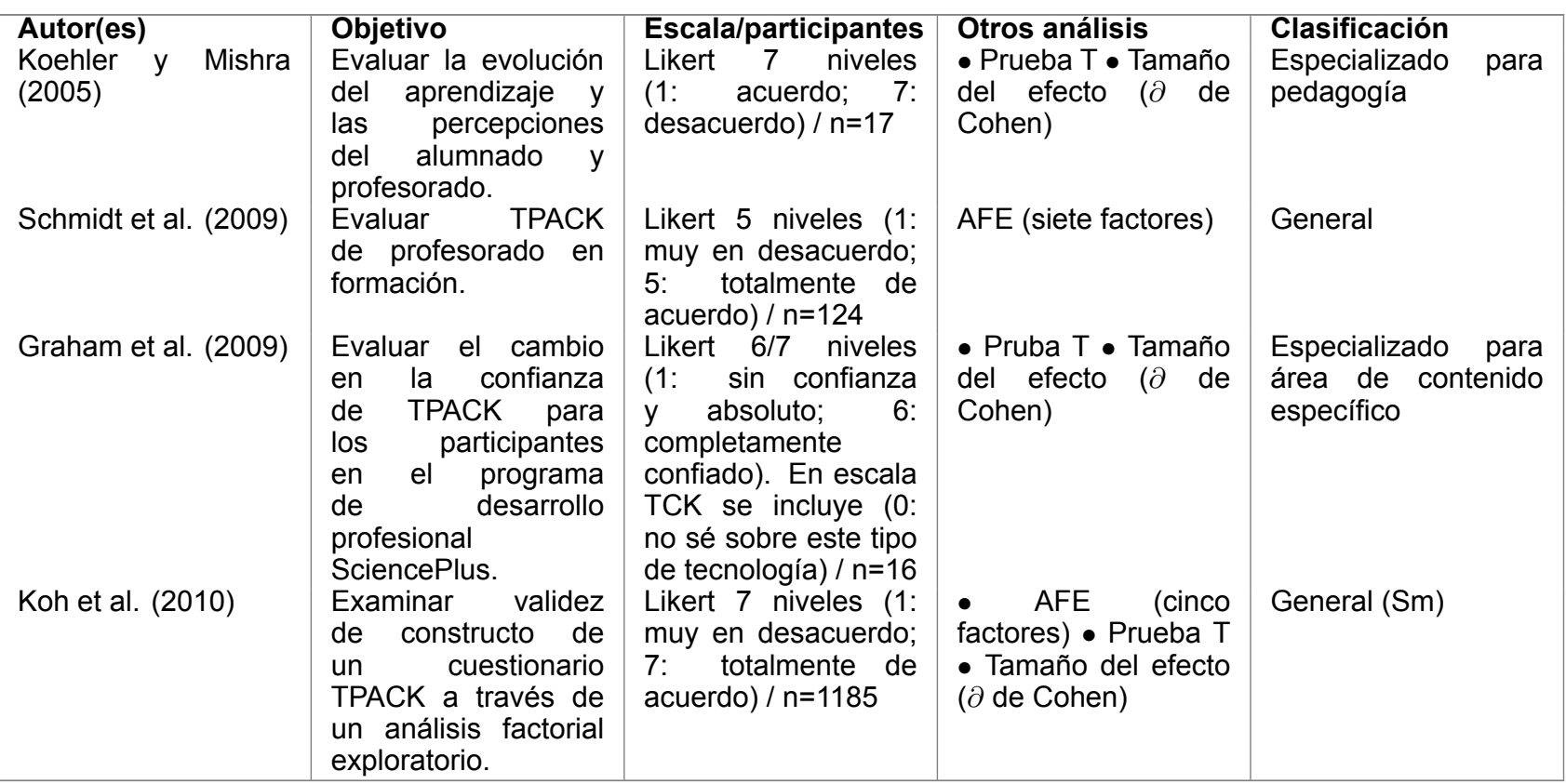


Chai et al. (2010)

Sahin (2011)

Kaya y Dag (2013)

\section{Jang y Tsai (2013)}

Lin et al. (2013)

Chai et al. (2013)

Valtonen et al. (2017)

\section{Table 2 continued}

Likert 7 niveles (1: muy en desacuerdo; 7: $\quad$ totalmente de acuerdo) $\mathrm{n}=439$ (curso previo) y $n=365$ (curso posterior) desarrollo percibido de los docentes en formación en términos de su TPACK.

Desarrollar un instrumento para que el profesorado evalúe sus conocimientos de TPACK.

Investigar estructura factorial mediante análisis factorial exploratorio y confirmatorio.

Desarrollar instrumento para determinar las creencias de autoeficacia del profesor de ciencias. Examinar el TPACK del profesorado en servicio de Ciencias.

Explorar las
percepciones del profesorado de ciencias sobre TPACK. Investigar perfil TPACK del profesorado de lengua china $y$ sus creencias pedagógicas.

Validar el instrumento TPACK-21 utilizando AFC.

Likert 5 niveles (1: nada; 5: todo) / $n=348$

un

Likert 5 niveles (1: nada; 5: todo) / $n=420, n=388$

Likert 5 niveles (1: muy en desacuerdo; 5: totalmente de acuerdo) / $\mathrm{n}=352$

Likert 5 niveles (1: no del todo cierto; 5 : muy cierto) / $n=1292$ Likert 7 niveles (1: muy en desacuerdo; 7: totalmente de acuerdo) / $n=222$

Likert 7 niveles (1: muy en desacuerdo; 7: totalmente de acuerdo) / n=287

Likert 6 niveles (1: necesito mucho conocimiento adicional sobre el tema; 6: tengo un gran conocimiento sobre el tema) / $\mathrm{n}=267$

el Likert 5 niveles (1: muy en desacuerdo; 5: totalmente de acuerdo) / $n=113$
- AFE (cuatro factores) - AFC - Chi-cuadrado Prueba T • Tamaño del efecto ( $\partial$ de Cohen) • Regresión lineal

- AFE (siete factores) - Validez discriminante

- AFE (diez factores) - AFC

- AFE (ocho factores) - AFC

- AFE (cuatro factores) - Prueba $T$ - ANOVA

- AFC (siete factores) - Prueba T

\section{- AFC (nueve} factores)

- AFC (seis factores)

Especializado para área de contenido específico

- AFE (tres factores) General
General (Sm)

General

General (Sm)

Especializado para área de contenido específico

Especializado para área de contenido específico

Especializado para área de contenido específico $(\mathrm{Sm})$

Especializado para área de contenido específico

Nota. AFE=Análisis Factorial Exploratorio; AFC=Análisis Factorial Confirmatorio

El cuestionario que se propone en este trabajo modifica al original en los componentes del CK, PCK, TCK, TPK y TPACK debido a que se especifican ítems sobre áreas de conocimientos que no son de interés para la investigación. Se eliminaron los ítems que no eran de Matemáticas (Ej. Tengo conocimientos suficientes sobre estudios sociales) y se añadieron otros sobre las competencias que debe poseer el profesorado de Matemáticas (Ej. Sigo desarrollos matemáticos recientes y sus aplicaciones, aunque no sean de mi principal campo de investigación). La escala Likert de cinco niveles se redujo a cuatro (1: nunca; 4: siempre). Además, se incluyeron en las características sociodemográficas de los participantes, el género, la experiencia docente 
y la experiencia con TIC.

Los ítems fueron revisados por seis expertos en tecnología educativa y Matemáticas para garantizar su redacción, claridad y adecuación. Los expertos realizaron 92 observaciones, de las cuales se aceptaron un $91.30 \%$. Las observaciones estuvieron dirigidas fundamentalmente a la mejora de redacción, claridad y adecuación de los ítems, ejemplos de estas modificaciones se encuentran en Tabla 3. Se desecharon 14 ítems por no existir consenso entre los expertos, quedando el cuestionario con 28 ítems (Tabla 4).

\section{Tabla 3}

Ejemplos de corrección ítems evaluados por expertos

\begin{tabular}{|c|c|c|}
\hline Observaciones & Redacción original & Redacción definitiva \\
\hline Redacción & $\begin{array}{l}\text { Puedo adaptar mi enseñanza a } \\
\text { diferentes estilos de aprendizaje del } \\
\text { alumnado. }\end{array}$ & $\begin{array}{l}\text { Adapto mi enseñanza a los diferentes } \\
\text { estilos de aprendizaje del alumnado. }\end{array}$ \\
\hline Claridad & $\begin{array}{l}\text { Sigo desarrollos matemáticos } \\
\text { recientes y sus aplicaciones. }\end{array}$ & $\begin{array}{l}\text { Sigo desarrollos matemáticos } \\
\text { recientes y sus aplicaciones, aunque } \\
\text { no sean de mi principal campo de } \\
\text { investigación. }\end{array}$ \\
\hline Adecuación & $\begin{array}{l}\text { Reflexiono sobre cómo las } \\
\text { herramientas tecnológicas podrían } \\
\text { tener un efecto en el aprendizaje de } \\
\text { las Matemáticas. }\end{array}$ & $\begin{array}{l}\text { Cambio de componente TCK a } \\
\text { TPACK. }\end{array}$ \\
\hline
\end{tabular}

Tabla 4

Cuestionario para medir TPACK en el profesorado universitario de Matemáticas

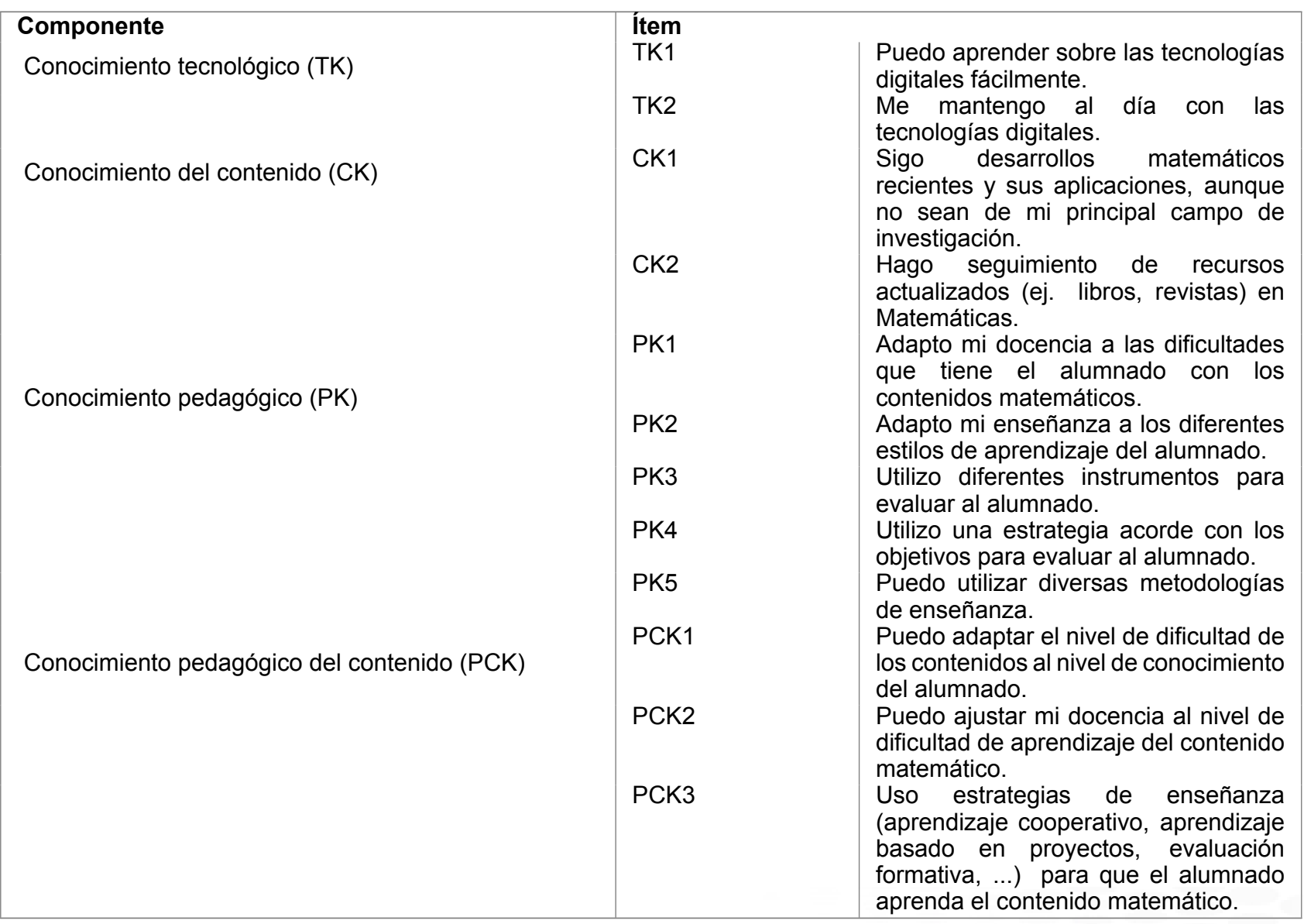




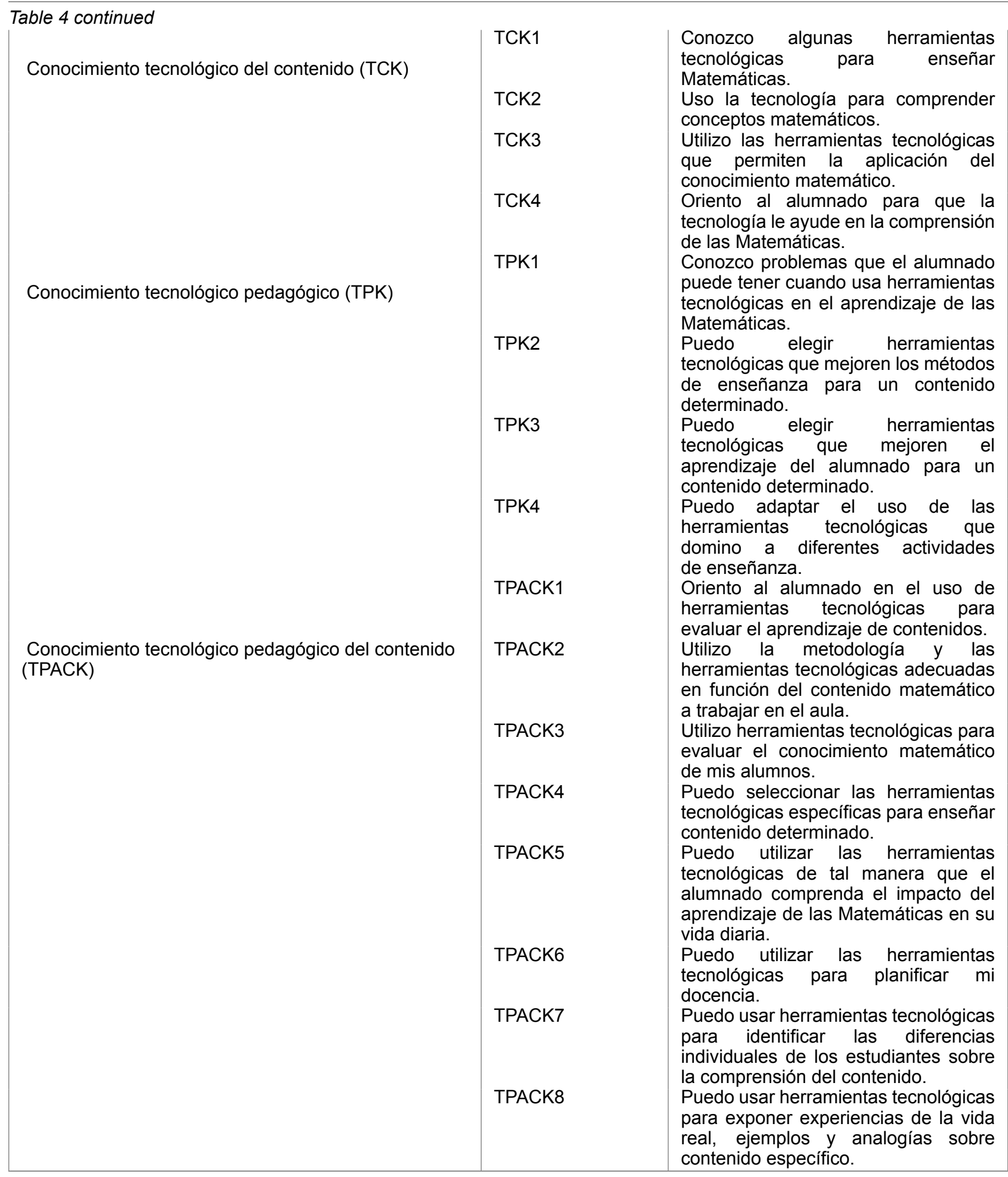

Una vez que se modificó el cuestionario original era necesario confirmar la validez y fiabilidad nuevamente. Con este fin, se utilizó el software AMOS versión 22, especializado en resolver problemas de modelado de ecuaciones estructurales, ampliamente utilizado por los investigadores para el análisis multivariante al integrar el uso de varios métodos como: regresión, análisis factorial, correlación y análisis de varianza (Thakkar, 2020). Se realizó un modelo de ecuaciones estructurales para asegurarnos que el cuestionario fuera apropiado con el fin de explorar el TPACK en el profesorado de Matemáticas con el modelo de siete factores. 
Identificado el modelo se realizó el análisis factorial confirmatorio (AFC). Para investigar si los componentes identificados se ajustaban a los datos se siguió la sugerencia de Kline (2015) sobre el conjunto mínimo de estadísticas de ajuste que deben informarse siempre que sea posible: a) modelo chi-cuadrado con sus grados de libertad y valor de $p, b$ ) error cuadrático medio de la aproximación de la raíz de Steiger-Lind (RMSEA) y su intervalo de confianza del $90 \%$, c) índice de ajuste comparativo de Bentler (CFI) y d) raíz normalizada media cuadrada residual (SRMR). En este sentido, Tabachnick y Fidell (2012) especifican que el CFI con valores superiores a .95 indica un excelente modelo de ajuste, de .90-.95 se considera un buen ajuste; el RMSEA con valores aproximados a .06 o menores es considerado muy razonable para un buen ajuste; y la SRMR con valores menores que .08 es aceptable.

\section{Resultados}

El modelo hipotético se presenta en la Figura 1, donde los círculos representan variables latentes y los rectángulos representan variables medidas. La ausencia de una línea que conecta las variables no implica un efecto directo hipotetizado. Se hipotetiza un modelo de siete factores: TK, CK, PK, PCK, TCK, TPK y TPACK. Los ítems TK1 y TK2, sirven como indicadores del factor TK; CK y CK2 como indicadores del factor CK; PK1, PK2, PK3, PK4 y PK5 como indicadores del factor PK; PCK1, PCK2 y PCK3 como indicadores del factor PCK; TCK1, TCK2, TCK3 y TCK4 como indicadores del factor TCK; TPK1, TPK2, TPK3 y TPK4 como indicadores del factor TPK; y TPACK1, TPACK2, TPACK3, TPACK4, TPACK5, TPACK6, TPACK7 y TPACK8 como indicadores del factor TPACK.

Los supuestos de normalidad y linealidad multivariantes se evaluaron a través del software SPSS 22. Se realizaron análisis de modelos de ecuaciones estructurales (SEM) utilizando datos de 183 docentes. No había datos faltantes.

Se utilizó la estimación de máxima verosimilitud para estimar todos los modelos. El modelo de independencia que prueba la hipótesis de que todas las variables no están correlacionadas fue fácilmente rechazable, $x^{2}(378, N=183)=8.63, p<.01$. El modelo hipotético se probó a continuación y se encontró soporte para el mismo, $x^{2}(329, \mathrm{~N}=183)=1.73, \mathrm{p}<.005$. La prueba de diferencia de Chi-cuadrado indicó la mejora significativa en el ajuste entre el modelo de independencia y el modelo hipotetizado. Los valores de $\mathrm{CFI}=.92$, RMSEA $=.06$ con un intervalo de $[.05 ; .07]$ y $\mathrm{SRMR}=.06$ son considerados como aceptables para un buen ajuste del modelo a los datos.

Por último, las correlaciones entre los componentes del modelo que se muestran en la Tabla 5 revelan que los coeficientes varían desde .21 (CK y TPK, CK y TPACK) hasta .90 (PK-PCK). El desarrollo del TPACK pudiera estar altamente influenciado por los componentes TK, PK, PCK, TCK y TPK. La fiabilidad de los siete factores en general es de .95, asimismo las puntuaciones de cada uno de los componentes en la Tabla 6.

\section{Discusión}

El cuestionario presentado en este trabajo es un aporte para examinar el TPACK del profesorado universitario de Matemáticas. Tiene como ventaja que se fundamenta en la necesidad de desarrollar cuestionarios específicos como identifican Chai et al. (2016) y Willermark (2018), además de permitir la medición del TPACK del profesorado en entornos de Educación Superior (Chai et al., 2016). También es posible obtener los puntajes de los docentes en cada componente del modelo, de forma tal que puedan realizarse comparaciones entre grupos o métodos que permitan relacionar variables.

Sobre la base del objetivo planteado en la investigación, luego de los análisis realizados y los resultados obtenidos, se puede destacar que el Cuestionario para medir el TPACK en el profesorado universitario de Matemáticas resulta válido y fiable. La prueba de Alfa de Cronbach, así como el AFC arrojan resultados resultados que evidencian la fortaleza del intrumento.

El tamaño de la muestra utilizada para confirmar la validez y fiabilidad del cuestionario, según Worthington y Whittaker (2006) es considerado como adecuado, permitió obtener resultados de que es una medida confiable de TPACK y sus dominios de conocimiento relacionados. En este sentido, investigaciones han confirmado la validez y fiabilidad de instrumentos para medir TPACK con tamaños de muestra suficientes (Bilici et al., 2013; Chai et al., 2010; Jang y Tsai, 2013; Kaya y Dag, 2013; Koh et al., 2010; Sahin, 2011;), adecuados (Chai et al., 2013; Lin et al.,2013; Valtonen et al., 2017) y pequeños (Cubeles y Riu, 2018; Schmidt et al.,2009). En trabajos futuros se puede refinar el instrumento mediante la obtención de un tamaño de muestra más grande. Las siete variables que mide el cuestionario están acordes con el modelo teórico TPACK y los resultados demuestran que el cuestionario mide los componentes con validez y fiabilidad. La eliminación de ítems mediante la revisión de los expertos permitió plantear un modelo hipotético (Figura 1) que tiene un buen ajuste 


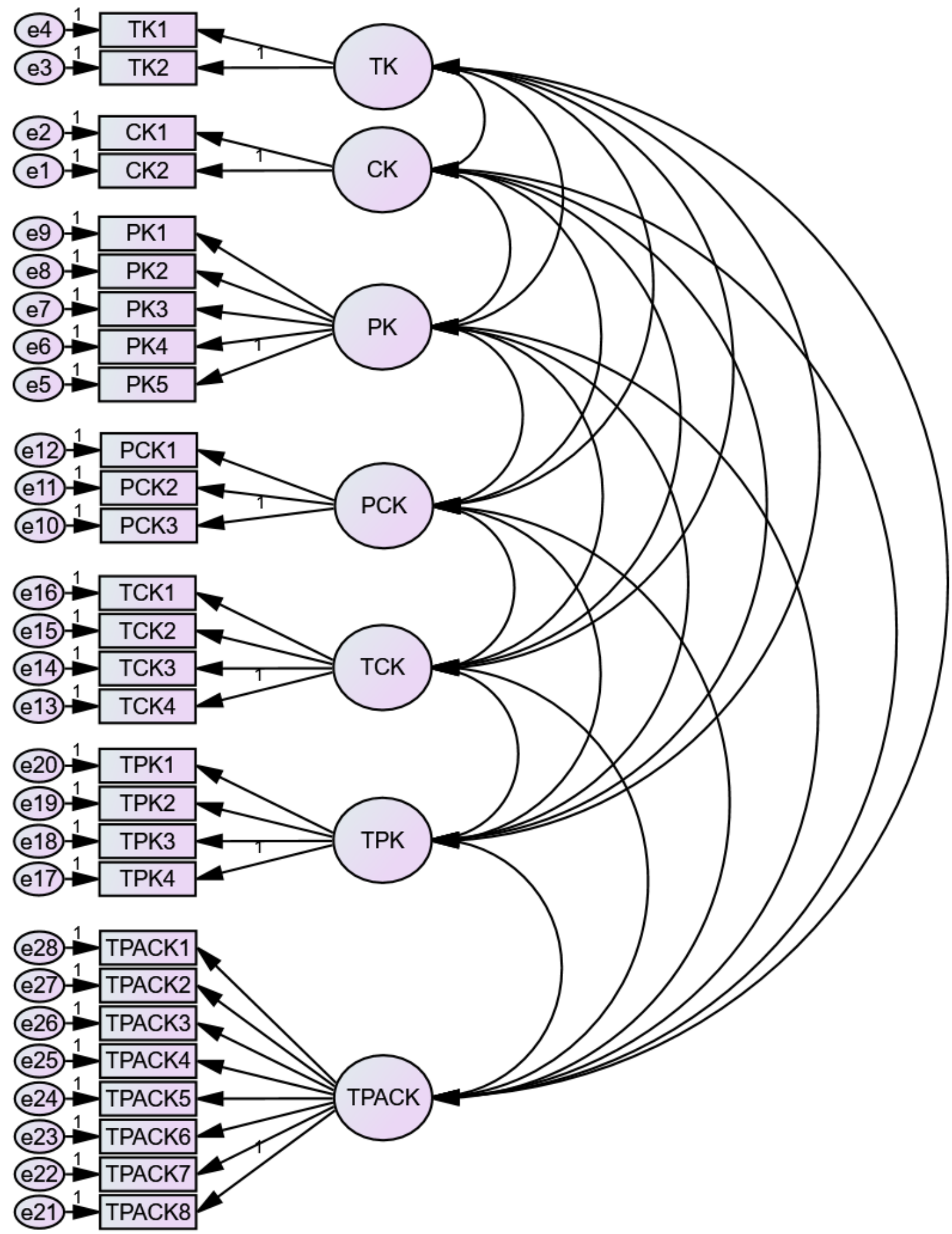

Figura 1. Modelo hipotético de AFC para componentes modelo TPACK. 
Tabla 5

Correlaciones entre componentes

\begin{tabular}{|c|c|c|c|}
\hline \multicolumn{3}{|c|}{ Correlación } & Estimado \\
\hline CK & $<->$ & TK & .31 \\
\hline CK & $<->$ & PK & .39 \\
\hline CK & $<->$ & PCK & .31 \\
\hline CK & $<->$ & TCK & .36 \\
\hline CK & $<->$ & TPK & .21 \\
\hline CK & $<->$ & TPACK & .21 \\
\hline TK & $<->$ & PK & .29 \\
\hline TK & $<->$ & PCK & .31 \\
\hline TK & $<->$ & TCK & .55 \\
\hline TK & $<->$ & TPK & .40 \\
\hline TK & $<->$ & TPACK & .48 \\
\hline PK & $<->$ & PCK & .90 \\
\hline PK & $<->$ & TCK & .55 \\
\hline PK & $<->$ & TPK & .53 \\
\hline PK & $<->$ & TPACK & 63 \\
\hline PCK & $<->$ & TCK & .58 \\
\hline PCK & $<->$ & TPK & .59 \\
\hline PCK & $<->$ & TPACK & .73 \\
\hline TCK & $<->$ & TPK & .82 \\
\hline TCK & $<->$ & TPACK & .87 \\
\hline TPK & $<->$ & TPACK & .87 \\
\hline
\end{tabular}

Tabla 6

Puntuaciones en componentes TPACK

\begin{tabular}{|l|l|l|l|l|}
\hline Componente & Media & SD & Mediana & Alpha \\
TK & 3.04 & .53 & 3.00 & .62 \\
CK & 3.06 & .67 & 3.00 & .80 \\
PK & 3.31 & .46 & 3.20 & .78 \\
PCK & 3.25 & .56 & 3.33 & .67 \\
TCK & 2.95 & .64 & 3.00 & .88 \\
TPK & 2.87 & .63 & 3.00 & .87 \\
TPACK & 2.68 & .67 & 2.63 & .92 \\
\hline
\end{tabular}

a los datos según la sugerencia de Kline (2015). A pesar de existir investigaciones (Bilici et al., 2013; Chai et al., 2010; Jang y Tsai, 2013; Kaya y Dağ, 2013; Koh et al., 2010; Valtonen et al., 2017) que presentan dificultades para demostrar instrumentos válidos que midan los siete componentes del modelo, esta investigación logra resultados similares a los de Schmidt et al. (2009), Sahin (2011) y Lin et al. (2013).

Los descriptivos sobre los componentes del modelo sugieren que el profesorado universitario de Matemáticas tiene elevados conocimientos sobre pedagogía (PK, PCK); sin embargo, ocurre de forma diferente con los conocimientos que incluyen tecnología (TCK, TPK, TPACK).

Con respecto a las correlaciones entre los componentes del modelo, los componentes pedagógicos sin tecnología (PK, PCK) tienen la más alta correlación. Del mismo modo ocurre en los resultados descritos por Cubeles y Riu (2018). Esto puede ser razonable porque el cuestionario está dirigido al profesorado universitario, donde se especializan en una disciplina. Dichos hallazgos implican que el profesorado universitario de Matemáticas percibe una mayor confianza en estos conocimientos para la integración de las tecnologías. En consecuencia, la alta correlación entre PCK y TPACK así lo demuestra (Tabla 5). Los resultados indican para los datos analizados en el estudio que la relación entre PK-CK es más fuerte que la relación entre TK-PK y TK-CK, este resultado se alinea con el estudio realizado por Valtonen et al. (2017).

Respecto a la aplicabilidad y la contribución al campo de estudios, es posible afirmar que el cuestionario permite obtener información válida y fiable sobre el conocimiento técnico, pedagógico y tecnológico del profesorado universitario de Matemáticas. Además, puede ser utilizado para analizar los conocimientos de los profesores 
según el modelo TPACK de modo que puedan desarrollarse programas de capacitación para contribuir a la integración de las tecnologías en las aulas. También es posible utilizar el instrumento para investigar cómo cambiaron los conocimientos de los profesores universitarios de matemáticas sobre la enseñanza y la tecnología durante un conjunto de cursos ofrecidos tanto presencialmente como en línea. Asimismo, puede aportar a investigaciones que establezcan relaciones entre creencias del profesorado y la integración de las tecnologías en la práctica. Futuros trabajos pueden aplicar el instrumento a muestras mayores y debería triangularse la información obtenida con otros tipos de instrumentos de medición como entrevistas y observaciones en la práctica docente.

\section{Conclusiones}

En conclusión, el cuestionario sobre conocimientos del profesorado universitario de matemáticas para la integración tecnológica es un instrumento con una alta consistencia interna, oscilando en sus dimensiones entre .62 y .92. Dichas dimensiones han sido validadas mediante un modelo de ecuaciones estructurales con un ajuste aceptable entre los datos y el modelo.

Los resultados de este estudio apuntan a varias áreas para futuras investigaciones. Primero, se pueden agregar más ítems de tipo TK y CK para fortalecer la medición de estos constructos. En segundo lugar, los estudios de validación de constructo se pueden realizar de varias formas. Por ejemplo, este estudio se puede replicar con profesores conocidos por ser integradores de tecnología ejemplares para determinar si podrían hacer distinciones claras entre los constructos TPACK. También se pueden realizar estudios comparativos de cuestionarios TPACK mediante la reproducción en diferentes países. En tercer lugar, es necesario seguir investigando la relación entre los constructos TPACK y las variables demográficas de los docentes, ya que permitirá mejorar la planificación de los programas de desarrollo docente. Finalmente, para futuras aplicaciones sería recomendable complementarlo con un estudio más profundo de carácter cualitativo, ya que las preguntas cerradas limitan las respuestas de los docentes. Asimismo, sería interesante conocer la satisfacción de los estudiantes con integración de las tecnologías en asignaturas de matemáticas.

\section{Referencias}

Agyei, D. D., y Voogt, J. M. (2015). Pre-service teachers' TPACK competencies for spreadsheet integration: insights from a mathematics-specific instructional technology course. Technology, Pedagogy and Education, 24(5), 605-625. Retrieved from https://dx.doi.org/10.1080/1475939x.2015.1096822 10.1080/1475939x.2015 .1096822

Alhashem, F., Alrwaished, N., y Alkandari, A. (2017). Exploring In- and Pre-Service Science and Mathematics Teachers' Technology, Pedagogy, and Content Knowledge (TPACK): What Next? EURASIA Journal of Mathematics, Science and Technology Education, 13(9), 6113-6131. Retrieved from https://dx.doi.org/10 .12973/eurasia.2017.01053a 10.12973/eurasia.2017.01053a

Baser, D., Kopcha, T. J., y Ozden, M. Y. (2016). Developing a technological pedagogical content knowledge (TPACK) assessment for preservice teachers learning to teach English as a foreign language. Computer Assisted Language Learning, 29(4), 749-764. Retrieved from https://dx.doi.org/10.1080/09588221.2015 $.104745610 .1080 / 09588221.2015 .1047456$

Bilici, S. C., Yamak, H., Kavak, N., y Guzey, S. S. (2013). Technological pedagogical content knowledge selfefficacy scale (TPACK-SeS) for pre-service science teachers: Construction, validation, and reliability. Eurasian Journal of Educational Research, 13(52), 37-60.

Chai, C. S., Koh, J. H. L., y Tsai, C. C. (2010). Facilitating preservice teacher's development of TPACK. Journal of Educational Technology \& Society, 13(4), 63-73.

Chai, C. S., Koh, J. H. L., y Tsai, C. C. (2013). A review of technological pedagogical content knowledge. Journal of Educational Technology \& Society, 16(2), 31-51.

Chai, C. S., Koh, J. H. L., y Tsai, C. C. (2016). A review of the quantitative measures of technological pedagogical content knowledge (TPACK). In M. C. Herring, M. J. Koehler, y P. Mishra (Eds.), Handbook of Technological Pedagogical Content Knowledge (TPACK) for Educators (Second Edition ed., p. 87-106). Routledge. Retrieved from https://doi:10.4324/9781315771328 https://doi:10.4324/9781315771328

Cubeles, A., y Riu, D. (2018). The effective integration of ICTs in universities: the role of knowledge and academic experience of professors. Technology, Pedagogy and Education, 27(3), 339-349. Retrieved from https://dx.doi.org/10.1080/1475939x.2018.1457978 10.1080/1475939x.2018.1457978

Fan, J., Peng, L., Li, K. H., y Tan, C. S. (2012). Wafer-level hermetic packaging of 3D microsystems with low-temperature $\mathrm{Cu}$-to-Cu thermo-compression bonding and its reliability. Journal of Micromechanics and Microengineering, 22, 105004-105004. Retrieved from https://dx.doi.org/10.1088/0960-1317/22/10/105004 $10.1088 / 0960-1317 / 22 / 10 / 105004$ 
Finger, G., Romeo, G., Lloyd, M., Heck, D., Sweeney, T., Albion, P., y Jamieson-Proctor, R. (2015). Developing Graduate TPACK Capabilities in Initial Teacher Education Programs: Insights from the Teaching Teachers for the Future Project. The Asia-Pacific Education Researcher, 24(3), 505-513. Retrieved from https://dx.doi.org/ 10.1007/s40299-014-0226-x 10.1007/s40299-014-0226-x

Giaconi, V., Perdomo-Díaz, J., Cerda, G., y Saadati, F. (2018). Prácticas docentes, autoeficacia y valor en relación con la resolución de problemas de matemáticas: diseño y validación de un cuestionario. Enseñanza de Las Ciencias. Revista de Investigación Y Experiencias Didácticas, 36(3), 99-99.

Graham, C. R., Burgoyne, N., Cantrell, P., Smith, L., St, C., Harris, L., y R. (2009). TPACK development in science teaching: Measuring the TPACK confidence of inservice science teachers. TechTrends, 53(5), 70-79. https://doi.org/10.1007/s11528-009-0328-0

Jang, S.-J., y Tsai, M.-F. (2012). Exploring the TPACK of Taiwanese elementary mathematics and science teachers with respect to use of interactive whiteboards. Computers \& Education, 59(2), 327-338. Retrieved from https://dx.doi.org/10.1016/j.compedu.2012.02.003 10.1016/j.compedu.2012.02.003

Kaya, S., y Dağ, F. (2013). Turkish adaptation of Technological Pedagogical Content Knowledge survey for elementary teachers. Educational Sciences: Theory \& Practice, 13, 302-306.

Kline, R. B. (2015). Principles and practice of structural equation modeling. New York, NY, USA: The Guilford Press.

Koehler, M. J., y Mishra, P. (2005). What Happens When Teachers Design Educational Technology? The Development of Technological Pedagogical Content Knowledge. Journal of Educational Computing Research, 32(2), 131-152. Retrieved from https://dx.doi.org/10.2190/0ew7-01wb-bkhl-qdyv 10.2190/0ew7-01wb-bkhl-qdyv Koehler, M. J., Mishra, P., Kereluik, K., Shin, T. S., y Graham, C. R. (2014). The technological pedagogical content knowledge famework. In J. M. Spector, M. D. Merrill, J. Elen, y M. J. Bishop (Eds.), Handbook of Research on Educational Communications and Technology (p. 101-111). Springer. https://doi:10.1007/ 978-1-4614-3185-5

Koh, J. H. L. H. L., Chai, C. S. S., y Tsai, C. C. C. (2010). Examining the technological pedagogical content knowledge of Singapore pre-service teachers with a large-scale survey. Journal of Computer Assisted Learning, 26(6), 563-573.

Lin, T. C., Tsai, C. C., Chai, C. S., y Lee, M. H. (2013). Identifying science teachers' perceptions of Technological Pedagogical and Content Knowledge (TPACK). Journal of Science Education and Technology, 22(3), 325-336. MISHRA, P., y KOEHLER, M. J. (2006). Technological Pedagogical Content Knowledge: A Framework for Teacher Knowledge. Teachers College Record, 108(6), 1017-1054. Retrieved from https://dx.doi.org/10.1111/ j.1467-9620.2006.00684.x 10.1111/j.1467-9620.2006.00684.x

Niess, M. L. (2011). Investigating TPACK: Knowledge Growth in Teaching with Technology. Journal of Educational Computing Research, 44(3), 299-317. Retrieved from https://dx.doi.org/10.2190/ec.44.3.c 10 .2190/ec.44.3.c

Reyes, V. C., Reading, C., Doyle, H., y Gregory, S. (2017). Integrating ICT into teacher education programs from a TPACK perspective: Exploring perceptions of university lecturers. Computers \& Education, 115, 1-19. Retrieved from https://dx.doi.org/10.1016/j.compedu.2017.07.009 10.1016/j.compedu.2017.07.009

Sahin, I. (2011). Development of Survey of Technological Pedagogical and Content Knowledge (TPACK). Turkish Online Journal of Educational Technology - TOJET, 10(1), 97-105.

Schmidt, D., Baran, E., Thompson, A., Mishra, P., Koehler, M., y Shin, T. (2009). Technological pedagogical content knowledge (TPACK): The development and validation of an assessment instrument for preservice teachers. Journal of Research on Technology in Education, 42(2), 123-149.

Shulman, L. (1986). Those who understand: Knowledge growth in teaching. Educational Researcher, 15(2), 4-14.

Shulman, L. (1987). Knowledge and teaching: Foundations of the new reform. Harvard Educational Review, 57(1), 1-23. 27.https://doi.org/10.17763/haer.57.1.j463w79r56455411

Tabachnick, B. G., y Fidell, L. S. (2012). Using multivariate statistics. New York: Harper and Row.

Thakkar, J. J. (2020). Structural Equation Modelling: Application for Research and Practice (with AMOS and R) (and others, Ed.). Springer. Retrieved from https://doi.org/10.1007/978-981-15-3793-6

Tseng, J. J. (2018). Exploring TPACK-SLA interface: insights from the computer-enhanced classroom. Computer Assisted Language Learning, 31(4), 390-412.

Valtonen, T., Sointu, E., Kukkonen, J., Kontkanen, S., Lambert, M. C., y Mäkitalo-Siegl, K. (2017). TPACK updated to measure pre-service teachers' twenty-first century skills. Australasian Journal of Educational Technology, 33(3), 15-31. Retrieved from https://dx.doi.org/10.14742/ajet.3518 10.14742/ajet.3518

Voogt, J., Fisser, P., Roblin, N. P., Tondeur, J., y van Braak, J. (2013). Technological pedagogical content knowledge - a review of the literature. Journal of Computer Assisted Learning, 29(2), 109-121. Retrieved from https://dx.doi.org/10.1111/j.1365-2729.2012.00487.x 10.1111/j.1365-2729.2012.00487.x

Willermark, S. (2011). Technological Pedagogical and Content Knowledge : A review of empirical studies published from. Journal of Educational Computing Research, 56(3), 315-343. 
Worthington, R. L., y Whittaker, T. A. (2006). Scale Development Research. The Counseling Psychologist, 34(6), 806-838. Retrieved from https://dx.doi.org/10.1177/0011000006288127 10.1177/0011000006288127

$\mathrm{Wu}$, Y. T. (2002). Research trends in technological pedagogical content knowledge (TPACK) research: A review of empirical studies published in selected journals from. British Journal of Educational Technology, 44(3), 20112014.

Zhou, Y., y Jin, M. (2017). The relationship between teachers online homework guidance and Technological Pedagogical Content Knowledge about educational use of web. The Asia-Pacific Education Researcher, 26(5), 239-247. https://doi:10.1007/s40299-017-0344-3 